\title{
Development and performance testing of a miniaturized multi-sensor system combining MOX and PID for potential UAV application in TIC, VOC and CWA dispersion scenarios
}

\author{
Francesca Fumian $^{1,2, a}{ }^{\mathbb{D}}$, Andrea Chierici ${ }^{1,3}$, Mattia Bianchelli $^{1,2}$, Luca Martellucci $^{1}$, \\ Riccardo Rossi ${ }^{1}$, Andrea Malizia ${ }^{4}$, Pasqualino Gaudio ${ }^{1}$, Francesco d'Errico ${ }^{3}$, \\ Daniele Di Giovanni ${ }^{1,5}$ \\ ${ }^{1}$ Department of Industrial Engineering, University of Rome Tor Vergata, 00133 Roma, Italy \\ 2 Joint NBC Defence School of Rieti, 02100 Rieti, Italy \\ 3 Department of Industrial and Civil Engineering, University of Pisa, 56122 Pisa, Italy \\ ${ }^{4}$ Department of Biomedicine and Prevention, University of Rome Tor Vergata, 00133 Rome, Italy \\ ${ }^{5}$ Unicamillus-Saint Camillus International University of Health Sciences, 00131 Rome, Italy
}

Received: 10 January 2021 / Accepted: 11 August 2021

(C) The Author(s), under exclusive licence to Società Italiana di Fisica and Springer-Verlag GmbH Germany, part of Springer Nature 2021

\begin{abstract}
The development of a tool to reduce the exposure of personnel in case of intentional or accidental toxic chemicals dispersion scenarios opens the field to new operational perspectives in the domain of operator safety and of critical infrastructure monitoring. The use of two sensors with different operating principles, metal oxide and photo-ionization detector, allows to confirm the presence of specific classes of chemicals in a contaminated area. All instruments are expected to be integrated into the payload of an unmanned aerial vehicle (UAV) and used for different purposes such as critical infrastructure surveillance focused on the volatile organic chemical and chemical warfare agents (CWA) detection and the post-incident of contamination level monitoring. In this paper, the authors presented the hardware set-up implemented and the test realized with CWAs simulants and will discuss the results obtained presenting advantages and disadvantages of this system in an application such as a UAV for the detection of chemical substances.
\end{abstract}

\section{Introduction}

Over the last few decades, the increase of threats associated to chemicals, and particularly to toxic industrial chemicals (TIC) derived from industry with their possible dual-use purpose, has led to the need for the development of new tools to detect and collect samples reducing or possibly eliminating the exposure of first responders inside the contaminated area. These solutions include the integration with unmanned aerial vehicle (UAV) with different detectors specifically targeted to chemical substances, whether warfare and not.

The employment of this equipment could meet both the needs of military personnel, in support of the Sampling and Identification of Biological, Chemical and Radiological Agents

a e-mail: francesca.fumian@esercito.difesa.it (corresponding author) 
(SIBCRA) team, and civilian emergency staff, involved in the monitoring of an industrial incident.

The UAV is integrated with the specific low-cost payload of this study and the overall system has to meet some basic requirements: high proximity flight, adequate payload, reliability, appropriate flight duration, low cost and proper maximum take-off weight (MTOW). In order to meet those specifications, the platform to support the mission will be a commercial rotary wing mini-drone (multirotor: quad or hexacopter), with an MTOW of $25 \mathrm{~kg}$, to avoid the necessity of a professional aircraft pilot. In several previous studies, this configuration has been proven to give the right balance between flight duration, payload and stability [1-5]. Few studies have been conducted on the use of gas sensors mounted on a drone [6] or have been developed and tested for the localization and mapping of VOC [1] or used for environmental monitoring and surveillance operations [2, 7-13].

Promptness is one of the crucial aspects in the monitoring of accidental or intentional release in the environment. Once the release has been noticed, indeed, several preparatory activities to grant access to the hotspot are needed. The accomplishment of those actions could last for hours or days and meanwhile may occur a loss of evidence about the dynamics of the accident, especially in the case of strongly volatile substances [14, 15].

Regarding the most suitable positioning of the sensors on the UAV, computational fluiddynamic simulations [16] are available to evaluate the influence that rotor turbulence had on the detection and identification capability and the best compromise between different configurations. A specific study on the best sensor location for the detection of particulate matter in case of a radioactive release had been already published [17]. A study is underway on evaluating the best position of sensors for chemical detection dispersion. In the wide range of available working principles for chemical detection, aiming at the payload integration with a UAV, two different technologies, metal oxide (MOX) and photo-ionization detector (PID), have been explored. By coupling those two working principles, it is possible to perform a qualitative assessment of the chemical class of the contamination and a quantitative assessment of its extent and entity. Indeed, the first operating principle has been chosen for its reliability in assessing concentration in the open air, its sensitivity, low weight and low cost. The second one has been signposted for its capability to perform identification, with a given certainty at least for the class of chemical substances.

In order to assess sensor detection capability in predicting the integration with a drone, selected detectors have been tested at different conditions with several VOCs with low toxicity, such as acetone, ethanol, ammonia, acetonitrile, etc. In this work are presented the results relevant to the detection of several CWAs simulants that are applicable for the extension to the corresponding real warfare agent. In order to assess the detection capability with CWAs simulants, the contaminants have been spread into the controlled volume of a glove box chamber, at a concentration comparable with substance limit of exposures (LOE), to understand whether the sensors limit of detection (LOD) is compatible with personnel exposition level (PEL) of CWAs and their simulants.

The paper is structured as follows: in Sect. 2 is given an overview of the different technologies explored to be applied to a UAV for a survey mission. Firstly, it discusses the state of the art of miniaturized sensors for chemical detection, such as MOX and PIDs to define a drone payload. Secondly, the architecture for their integration to the drone and data processing and transmission is described. Section 3 describes the experimental set-up of the test campaign conducted to assess the capabilities of the technologies selected and the CWAs simulant chosen for the trials. In Sect. 4, pieces of evidence of the results obtained are illustrated and discussed. Finally, in Sect. 5, regarding conclusions and future developments, the path for the integration of the whole system is defined. 


\section{Materials and methods}

In this section of the paper, techniques devoted to the implementation of specific functions in the overall integrated system proposed, such as detection by miniaturized environmental sensors, monitoring and data processing and transmission, were defined and described.

\subsection{Metal oxide (MOX) detectors}

MOX detectors, also known as electronic noses, are devices which mimic the functions of the human nose through a series of gas sensors combined with automatic learning algorithms. These sensors, which change their conductivity to gas exposure, are generally used for monitoring air quality (e.g., fire emissions, industrial VOCs). This family of devices generally consists of a single material and is characterized by:

- a high detection sensitivity (low LOD) of gaseous contaminants in air (it can detect hydrogen at a level of a few ppm);

- low power consumption (a heating current of only $42 \mathrm{~mA}$ ), weight and size;

- long technical life and low cost;

- use of simple electrical circuits.

The sensing element is a layer of semiconductor metal oxides deposited on an alumina substrate which, when coupled to the integrated heater that brings the sensing surface from 150 to $400{ }^{\circ} \mathrm{C}$ locally, forms the sensing chip (Fig. 1). Once exposed to a detectable gas, the conductivity of the semiconductor layer, oxidizing the target gas, changes proportionally with the concentration of the compound in the air. The conductivity change signal thus produced can be converted, through a simple electrical circuit, that measures the current that flows between working electrodes into an output signal corresponding to the gas concentration. Besides, an external circuit is needed to measure, amplify and acquire signals other than for the voltage control across the electrodes and heater of the MOX [19-21].

However, this class of sensors is not particularly selective for different gases and also suffers from problems such as long-term stability and environmental interference. However, recent studies have shown that, by using a combination of MOX materials, both selectivity and sensitivity, key aspects of sensor performance can be improved [19-22].

Specific MOX gas-sensitive layer reacts to oxidizing gases with increasing of the layer resistance and to reducing gases with decreasing of the layer resistance. The metal oxide chosen is different (electron acceptor/donor) depending on the substance to detect (electron donor/acceptor). Depending on the type of gas and the sensor type, a specific MOX gas sensor reacts in a concentration from a few ppb up to ppm. The most significant reason for the choice

Fig. 1 Metal oxide working principle scheme [18]

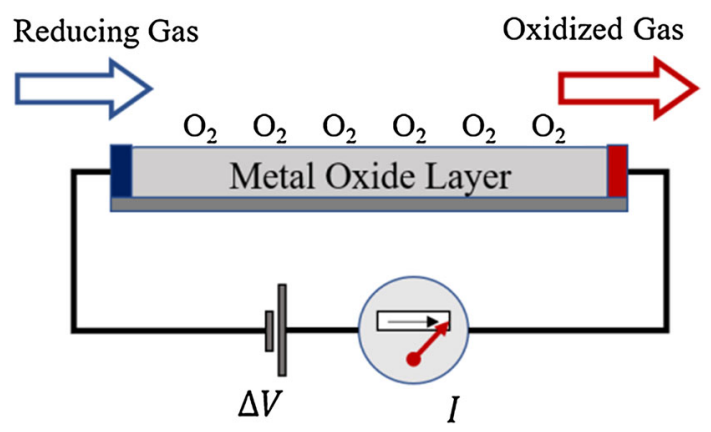


Fig. 2 General schematic of a photo-ionization detection sensor [26]

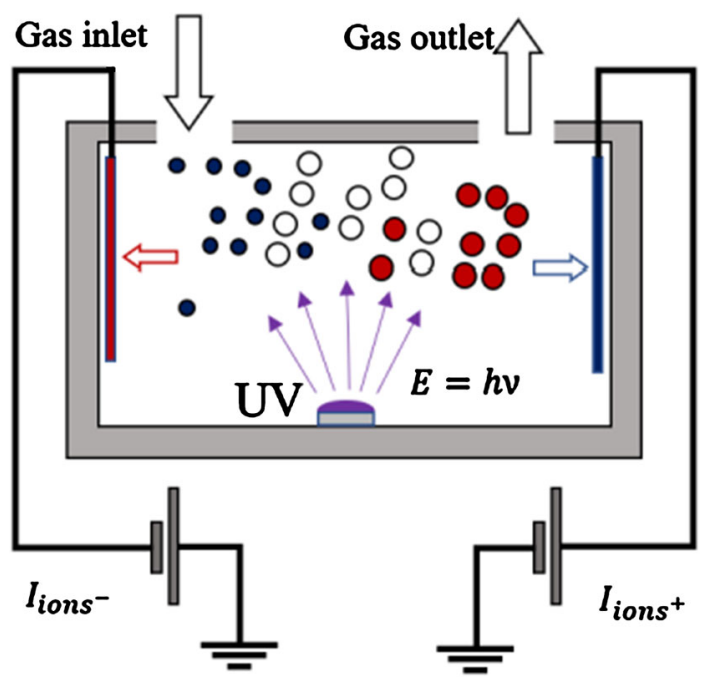

of MOX gas sensors to perform a preliminary analysis infield in case of contamination lies in the low cost and fast deployment of this kind of sensor.

\subsection{Photo-ionization detectors (PIDs)}

The second working principle selected for this application is photo-ionization detection. This principle is commonly applied to detect and monitor numerous hazardous substances, and instruments based on this method are relatively low cost and provide a quick response [23]. Compared to several other methods of detecting dangerous gases available on the market, PIDs enclose a combination of compact size, ease of use and maintenance, response rates and low LOD for several agents, including most volatile organic compounds (VOCs) and chemical warfare agents (CWAs) [24]. PID consists of a short-wave-length ultraviolet lamp shining inside a chamber, where is located a couple of electrodes with an applied potential. PIDs use the working principle of Cas ionization. When the sample gas reaches the chamber and is ionized by the PID lamp energy, the gas molecule gets excited, and its potential energy is altered and the substance is considered ionized (Fig. 2). If the energy is enough, the compound loses an electron $\left(\mathrm{e}^{-}\right)$and becomes a positive ion [25]. By measuring the current produced from the ionized compound, it is possible to obtain its concentration as parts-per-million (ppm) [24].

Ionization is possible for most substances; for some it is easier than for others. The aptitude of a substance to be ionized is its ionization energy (IE), measured with an energy scale in electron-volt (Ev). This scale generally ranges from a value of 7 to a value of about 16; the higher the value of IE, the stronger will have to be the lamp energy to be applied to ionize the substance.

The lamp chosen for this application is an Alphasense detector (PID-AH2) with a specific voltage of $10.6 \mathrm{eV}$, which is, therefore, able to ionize substances that have an IE lower than its specific voltage and detect them with a LOD of ppbs.

PIDs do not have identification capability: for a complete analysis (quantitative and qualitative), they have to be used in combination with an identification instrument. Nonetheless, if the monitoring occurs in a site where the involved chemicals are known from open sources or 


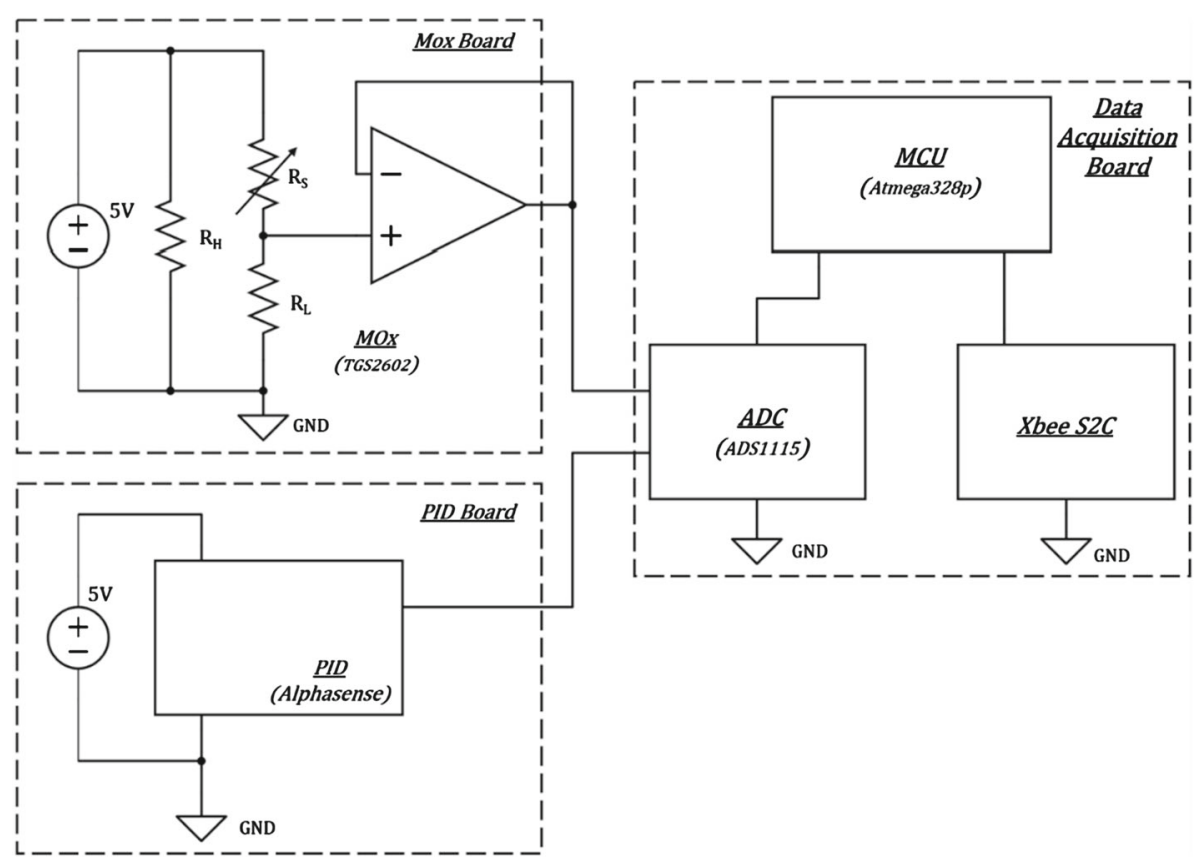

Fig. 3 General architecture of the readout system

information gathered from the site responsible, a quantification of detected substance can be done by using the available databases of the response factor $(\mathrm{RF})$, that is compound specific $[27,28]$.

Few applications have been previously conducted with the integration of those sensors with UAV; most of them devoted to combustion products [8,29] or particulate matter [9] investigation. For what regards industrial pollutants, evidence has been found of studies using electrochemical sensors [10], less sensitive than PID to VOC.

To acquire, process and transmit the electrical signal coming from the PID with a complete low-cost, low-energy demand and lightweight microcontroller, solution has been developed for the present experimental application, with the additional feature to use in parallel an array of different detectors at the same time, with single hardware in command.

\subsection{System integration and data processing and transmission}

The electronics front-end for the conditioning and acquisition of the MOX signals consists of two parts: a PWM constant current sink coupled to the heating resistor of the MOX and a 15-bit (single-ended) delta-sigma ADC (ADS1115, from Texas Instruments) which converts the MOX signal (resistor divider voltage) to digital values. The load resistor for the MOX has been set to $1 \mathrm{Kohm}$. Since PID sensors are already provided with an evaluation board, they are digitized directly by the ADC and no further control electronics are required for their conditioning. Digitized data are sent directly to a laptop through a radio frequency Xbee S2C module for further processing, visualization and logging. The general architecture of the readout system is described in Fig. 3, while in Fig. 4 the assembled prototype is shown. 

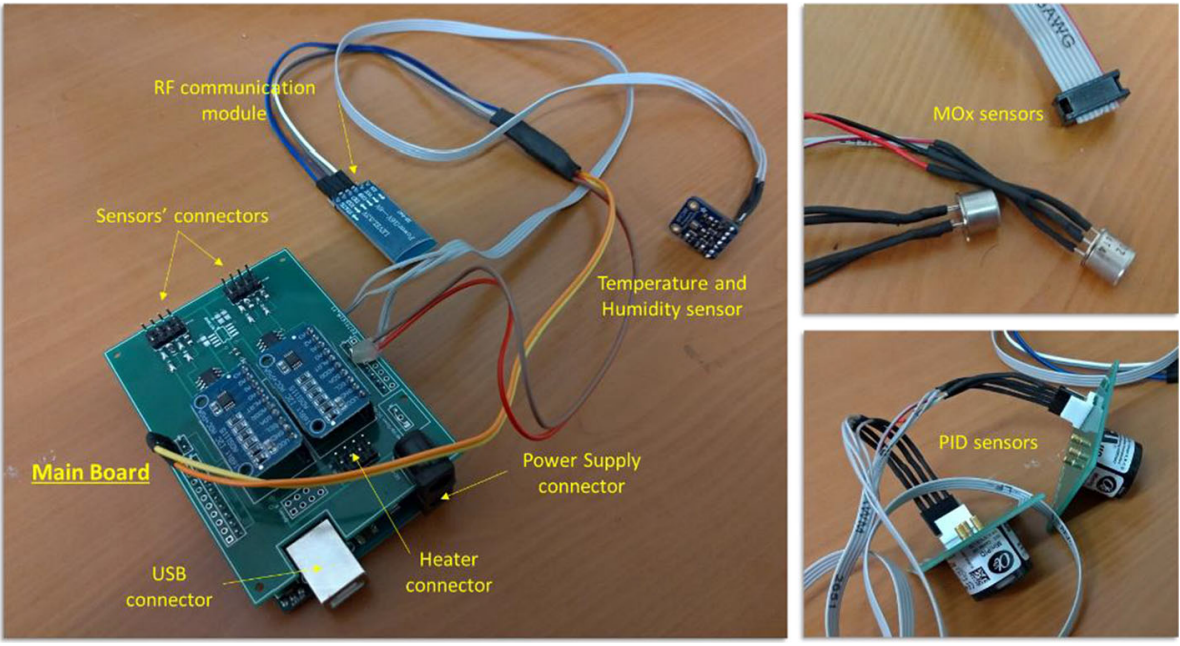

Fig. 4 Assembled prototype picture including components description

The nominal heating current to drive the MOX is dependent on the specific sensor chosen, which is, in this case, corresponds to $56 \pm 5 \mathrm{~mA}$ (TGS2602, Figaro Sensors), and it can be completely turned off $(0 \mathrm{~mA})$ or on $(56 \mathrm{~mA})$. The onboard programmable gain amplifier (PGA) of the ADC chosen allows for the scaling of the dynamic range for input signals between $4096 \mathrm{mV}$ and $0.256 \mathrm{mV}(\times 1$ to $\times 16)$ to investigate the response to chemical agents showing a different sensitivity in the sensors without modifying the hardware.

\section{Experimental tests campaign}

This section presents the experimental set-up of the tests conducted to verify the functionality and effectiveness of the multisensor system, identified as features for punctual monitoring and integrated with data processing and transmission unit. The tests should be intended as the first phase of a wider testing campaign, which will lead, step by step, to assess the functionality of the detection system as a whole.

\subsection{Multisensor system laboratory set-up}

The tests have been conducted in a controlled environment, the BlueThree 4' hood from Blueair, a hermetically sealed glove box with a Class III hood. Fresh air is introduced through a HEPA filter on the side and then expelled through a double HEPA filter system located on the top of the hood (Fig. 5), ensuring a negative pressure on the internal environment.

The hood, being gas-tight, guarantees the highest level of personal protection and is suitable for high-risk work, such as employing group 3 biological agents or high toxicity chemical agents. Moreover, it is fitted with sleeve butyl-rubber gloves, incorporated in the front glass panel, which ensure a total barrier between the operator and the worktop.

Once inside the hood, samples have to be maintained sealed one from the other to avoid the possible risk of cross-contamination due to turbulent airflow inside the cabin. 
Fig. 5 BlueThree 4' BSL III glove box
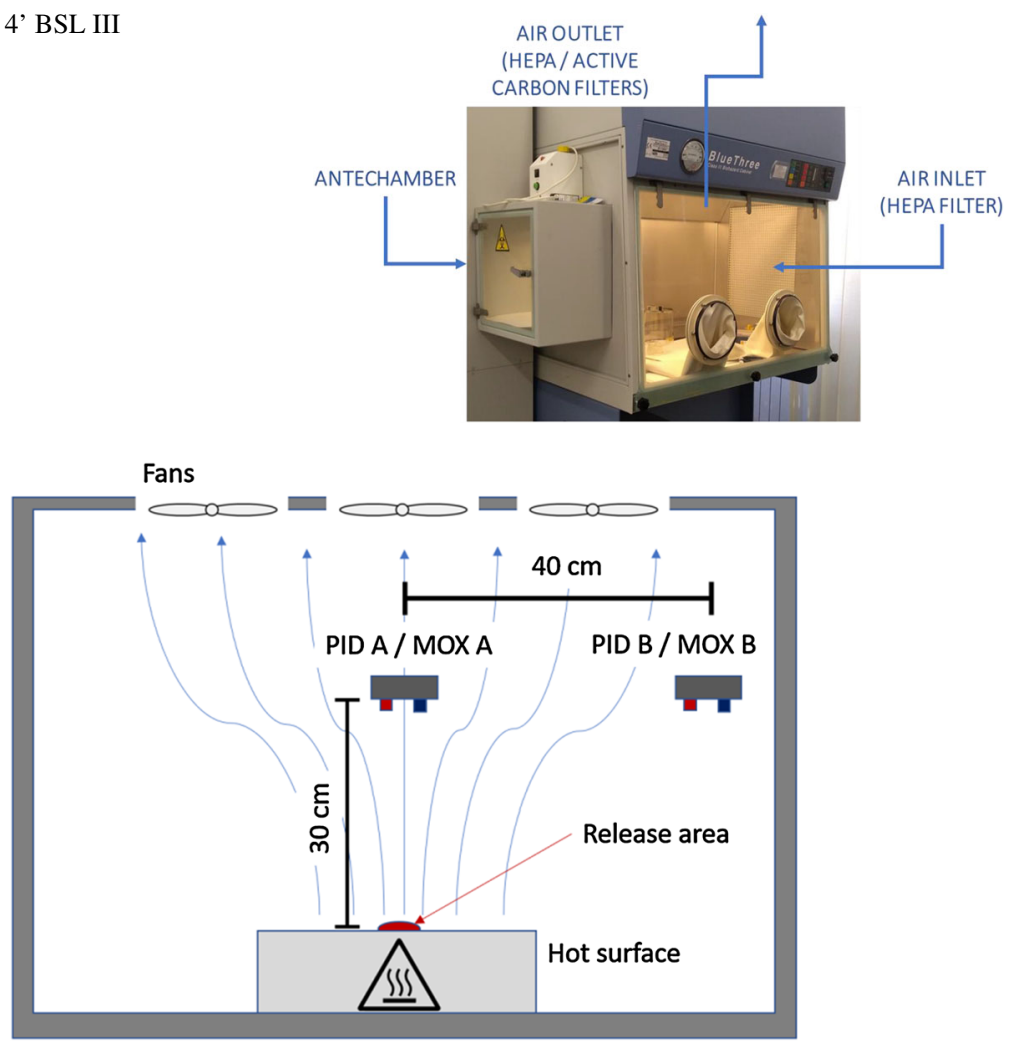

Fig. 6 Glove box experimental set-up

For this experimental test campaign, two sensors of each type have been employed, coupling one MOX and one PID in approximately the same position to expose them at the most similar possible contamination. The "A" couple of sensors is located on the vertical of the drop at $30 \mathrm{~cm}$ from the plate, and the "B" couple is located at $40 \mathrm{~cm}$ aside from couple " $\mathrm{A}$ " at the same relative height from the plate. Pressure and temperature inside the glove box were not controlled and remained for the whole duration of the tests at the average value of $T=$ $(26 \pm 1){ }^{\circ} \mathrm{C}, P=(970 \pm 10)$ mbar, humidity $=(44 \pm 2) \%$.

As shown in Fig. 6, the contamination has been created by depositing on a heating plate $\left(70{ }^{\circ} \mathrm{C}\right)$ the desired amount of pure substance as a drop, withdrawn with a GC/MS syringe, to reduce the delay due to the agent evaporation and obtain a more distinct signal.

\subsection{Chemical Warfare Agents (CWA) Simulants}

Generally speaking, in chemistry, a simulant of a component is a molecule resembling the original one in the structure and/or function. In the particular case of chemical warfare agents (CWA), a simulant must:

- be chemically similar to the real agent to activate the same response channels that the corresponding CWA activates in the detector;

- simulate the environmental behaviour (i.e., appearance, persistence, tissue penetration) of the real agent; 
- not have the same toxicity effects;

- not present the same related management issues (acquisition, transport, storage) of the real agent, as per consistent regulations (i.e., Chemical Weapon Convention (CWC), European Commission Dual-Use Regulation).

For this experimental test, campaign has been chosen to use simulants for three main classes of CWAs:

1. blister agents: to simulate sulphur mustard agent has been chosen the 2-chloro-ethylethyl-sulphate (2-CEES) [30], also known as half-mustard for its chemical structure.

2. nerve agents class B: to simulate sarin (GB) has been chosen its precursor di-methylmethyl-phosphonate (DMMP), a chemical listed in Schedule 2 of the CWC because it poses significant risks for its toxicity and it is not produced in large commercial quantities as an industrial commodity;

3. nerve agents class A: to simulate tabun (GA) has been chosen paraoxon, an organophosphate widely used in the past years as an insecticide in agriculture. As the real CWA, it has strong acetylcholinesterase-inhibiting behaviour.

All the chemical components were purchased from Sigma-Aldrich MERCK. In Table 1 are reported the main characteristics interest of this application of the simulant involved and relevant real CWAs, such as chemical formula, ionization energy (IE) and several exposure limits defined from the several responsible agencies as:

- PEL: permissible exposure limit (OSHA);

- IDLH: immediately dangerous to life and health (NIOSH) is usually a time-weighted average (TWA) limit over a time-shift;

- STEL: short-term exposure limit (ACGIH), over a short lapse of time, is usually $15 \mathrm{~min}$ as long as the time-weighted average is not exceeded;

- LCt50: median lethal dose (FDA) is the dose that is lethal for $50 \%$ of the population exposed to a certain concentration for a certain time;

- LD50: median lethal dose (FDA) is the dose that is lethal for 50\% of the population and is expressed as the mass of substance administered per unit mass of the test subject.

\section{Results and discussion}

One of the main objectives of this preliminary test campaign was to evaluate the dynamic response to different chemical agent categories and take confidence with the sensor qualitative outcome. In the test conditions described in the previous section, due to the evaporation of the contaminant under the turbulent flow of the hood, it has been difficult to assess the actual contaminant concentration at which each sensor was exposed.

During each measurement, to assess a blank measure of a baseline signal of an essentially clean environment, a drop of the contaminant was deposited on the heating plate after $20 \mathrm{~s}$ from the starting of the data acquisition, and each recording lasted approx. $2 \mathrm{~min}$. For every test, data analysis was conducted to evaluate the relative increment of the signal from its baseline; such processing involved the subtraction from the response signal of the mean value of the offset first and then the division of the result by the mean of the obtained blank value as shown in Eq. 1:

$$
\text { Relative counts }=\frac{\text { Counts }- \text { mean }(20 \text { s blank })}{\text { mean }(20 \text { s blank })}
$$




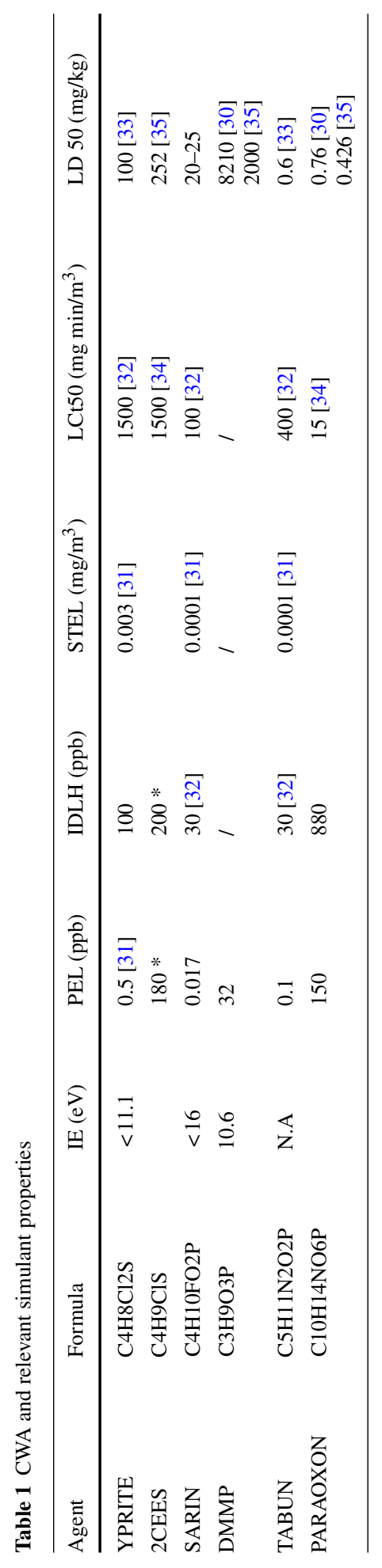



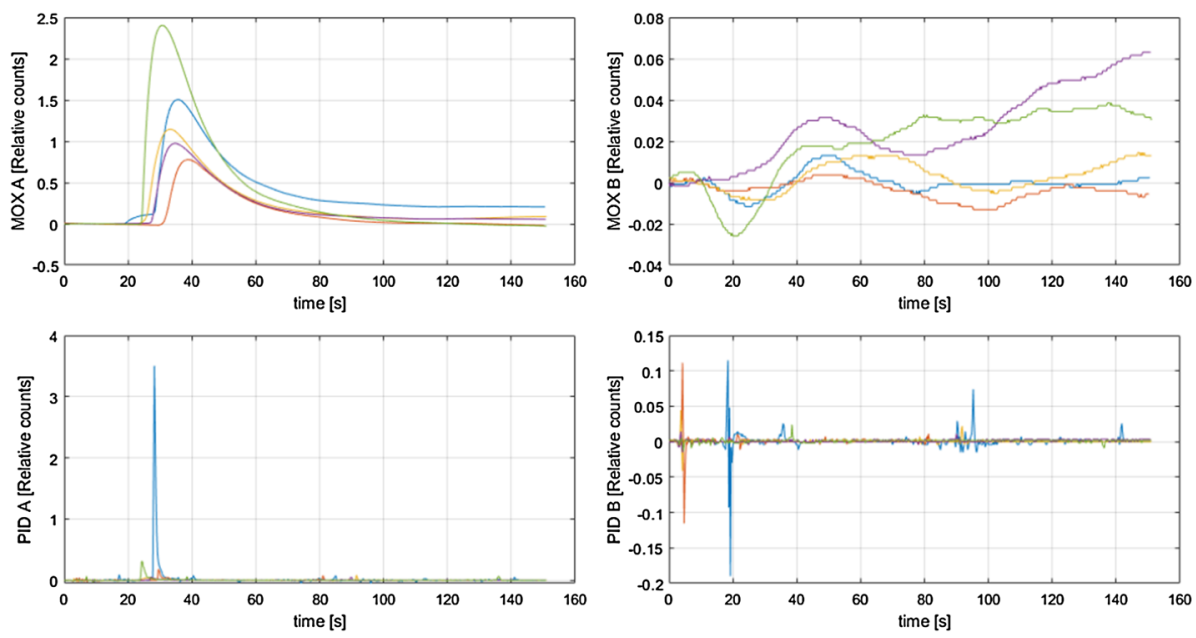

Fig. 7 MOX and PID response to the contamination with $1 \mu 1$ drop of 2-CEES (test repeated 5 times)

In this way, the overall counts were considered only caused by the actual contamination and not by environmental pollutants or by a drift of the sensor base signal. According to such an approach, the concept of a threshold alarm was introduced, and only a significant deviation from the environmental baseline (at least 2 times the basis) should be considered as an alarm.

From Figs. 7, 8, 9, 10, 11, 12, 13, 14, the experimental results are presented. On the left of each figure, the relative counts are inherent to a couple of MOX/PID sensors labelled as "A", which were the ones located on the vertical axis of the contamination. On the right of each figure, the relative counts are instead inherent to a couple of MOX/PID sensors labelled as "B", which were the ones located at $40 \mathrm{~cm}$ aside from the vertical axis of the contamination.

\subsection{Blister agent (sulphur mustard, HD) simulant tests}

The 2-CEES has the aspect of a moderately viscous, slightly yellow liquid, and it is characterized by an unpleasant onion smell; moreover, it is immiscible with water $(1062 \mathrm{mg} / \mathrm{L})$.

In Figs. 7, 8, and 9 the relative counts are reported, and they are inherent to the contamination obtained by pouring a certain volume, respectively, of $1 \mu 1,5 \mu 1$ and $10 \mu 1$, of 2-CEES corresponding to a maximum local concentration of about $4 \mathrm{ppm}, 20 \mathrm{ppm}$ and $40 \mathrm{ppm}$. Sensor response has been analysed as described in the introduction of Sect. 4. For each amount of simulant volume, the test has been repeated 5 times.

Regarding the sensors located on the vertical axis, the response in terms of multiples of the baseline blank is more significant for PID A than for MOX A; such effect becomes more evident with growing concentrations. This behaviour was expected because of the non-linear response of MOX to concentration changes (see Sect. 4.4-discussion). After the measurement, it can be observed how the signals from both MOX A and PID A go back to the baseline value; such behaviour suggests not only that the sensors are back to the original steady state, but also that the local volume can be considered clean, and that every new acquisition started from the same blank value. In Fig. 10 the overall relative counts for the three arrays of tests, showing the relevant standard deviation (SD), are presented. The SD has been calculated, for each of the three arrays of tests ( 5 repetitions at $1 \mu 1,5 \mu 1$ and $10 \mu 1$ 

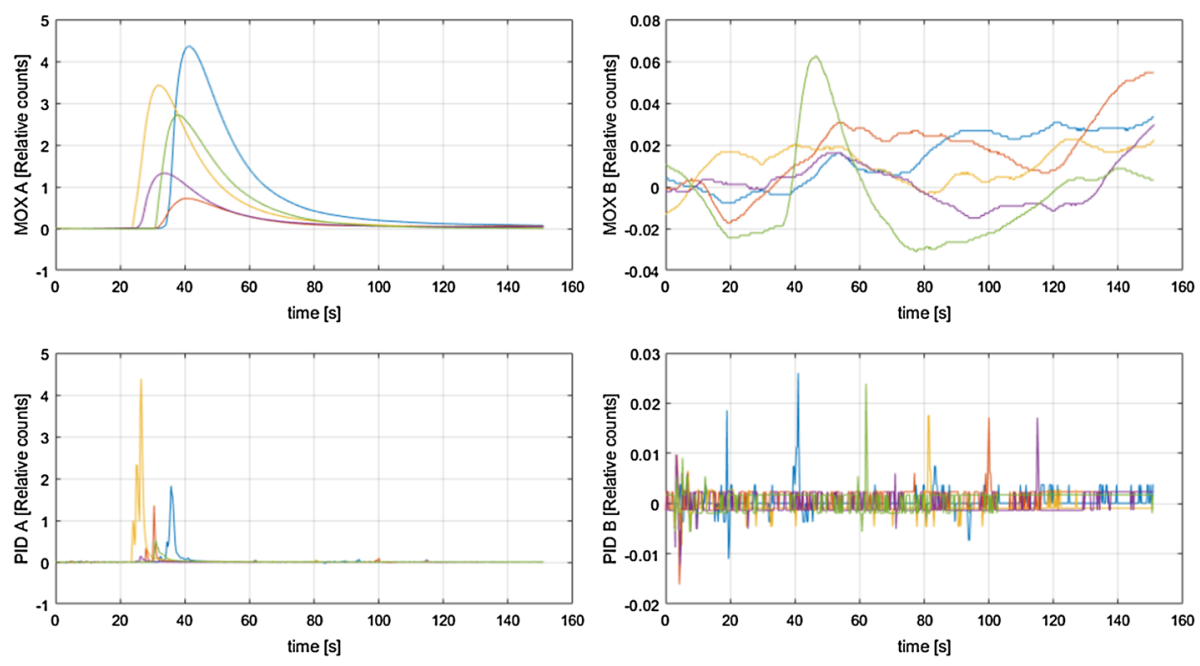

Fig. $8 \mathrm{MOX}$ and PID response to the contamination with $5 \mu 1$ drop of 2-CEES (test repeated 5 times)
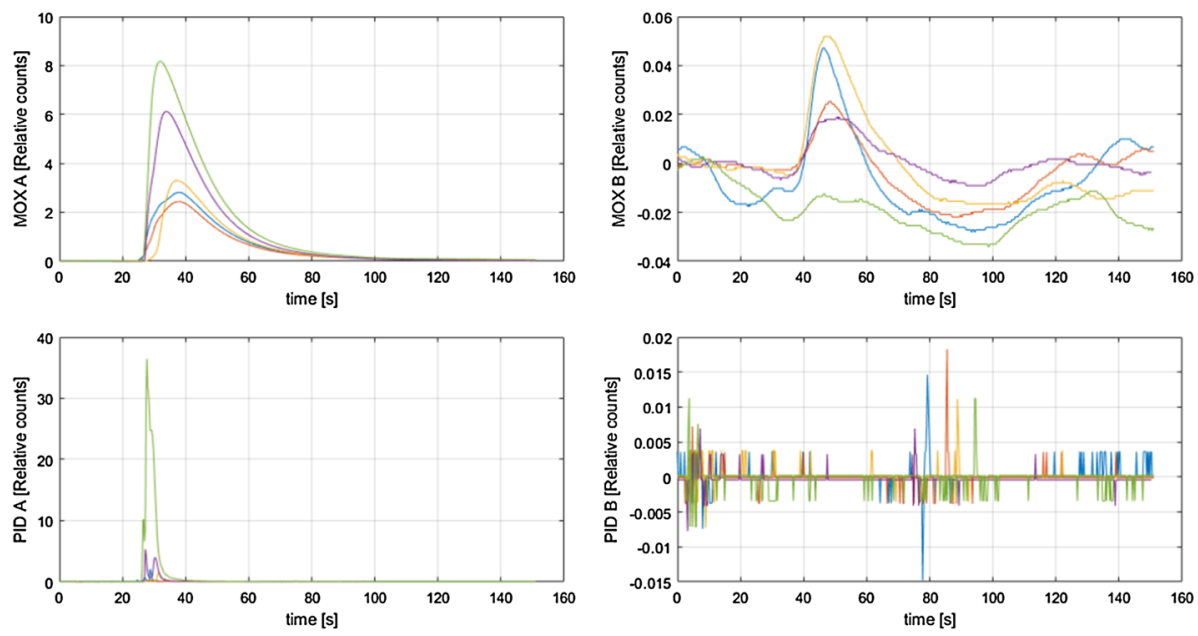

Fig. 9 MOX and PID response to the contamination with $10 \mu 1$ drop of 2-CEES (test repeated 5 times)

of agent volume), as the deviation from the arithmetic mean value of the measured counts, calculated at given intervals of time. To make the PID A response more readable, data are shown on a logarithmic scale.

Regarding PID B and MOX B, they were exposed to diluted contamination due to the aside position from the vertical axis and to the turbulent flow induced by the hood aspirator. With this premise, the relative counts of those two sensors resulted in a lower concentration and an irregular signal. However, it can be observed how the MOX signal shape, showing a peak and a relaxation slope is similar to the one obtained with the MOX placed in A position, but with a peak delay of $20 \mathrm{~s}$ due to the diffusion of the chemical. 

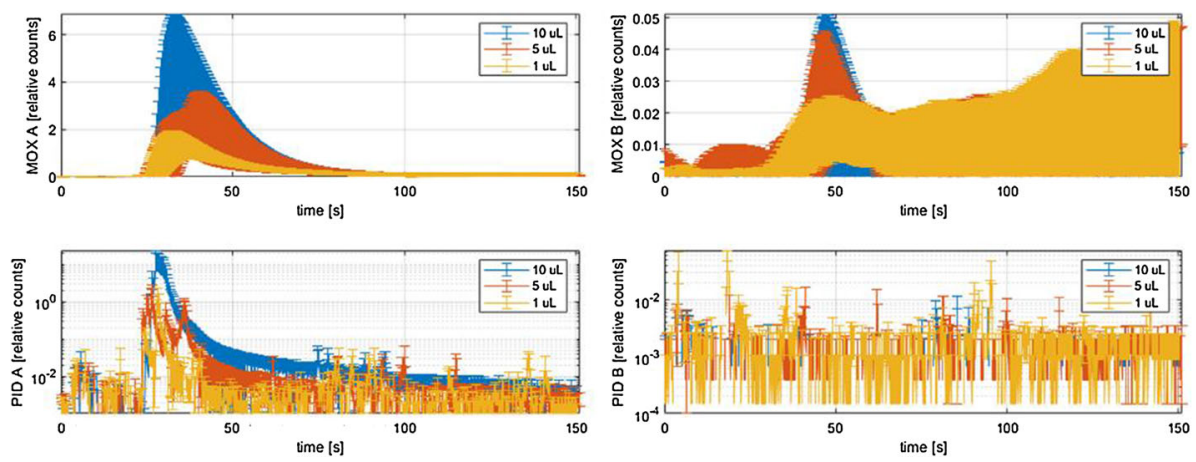

Fig. 10 MOX and PID overall responses with standard deviations to the contamination with $1 \mu 1,5 \mu 1$ and $10 \mu 1$ drop of 2-CEES
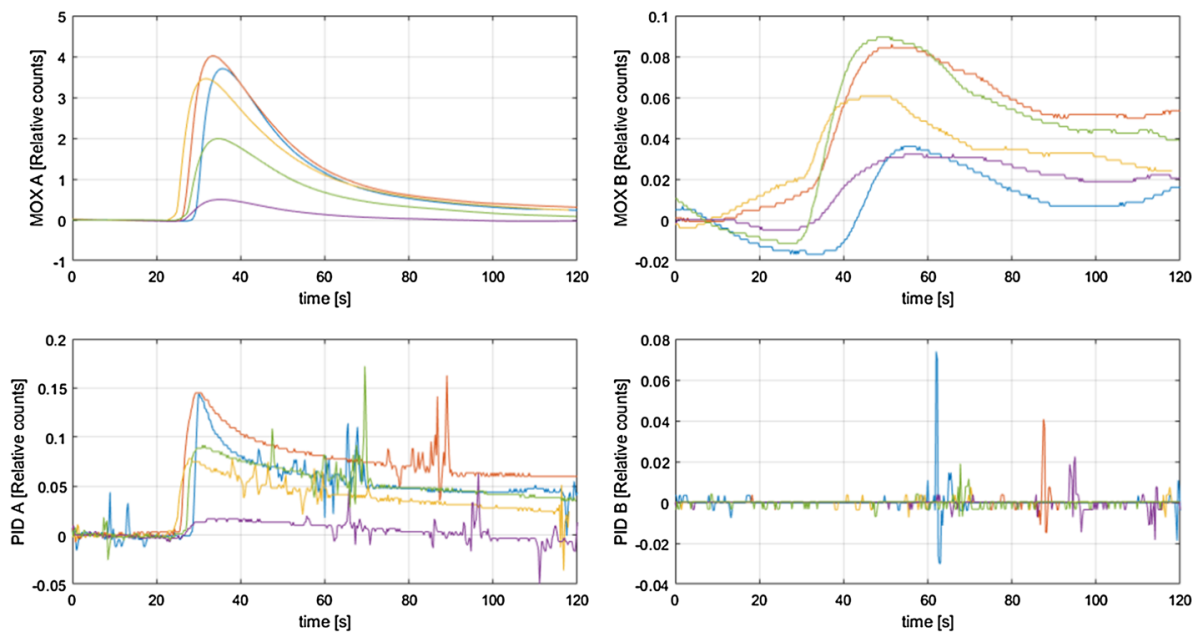

Fig. 11 MOX and PID response to the contamination with $10 \mu 1$ drop of DMMP (test repeated 5 times)

\subsection{Volatile nerve agent (sarin, GB) simulant tests}

DMMP has the aspect of a moderately viscous, transparent liquid, and it is characterized by an unpleasant onion smell; it is also soluble in water $\left(1 \times 10^{6} \mathrm{mg} / \mathrm{L}\right)$.

Figures 11 and 12 show the relative counts measured in response to the contamination obtained by pouring a certain volume, respectively, of $10 \mu 1$ and $100 \mu 1$, of DMMP corresponding to a maximum local concentration of about $45 \mathrm{ppm}$ and $450 \mathrm{ppm}$. Sensor response has been analysed as described in the introduction of Sect. 4. For $10 \mu 1$ of DMMP volume, the test has been repeated 5 times, while for $100 \mu$ l of DMMP volume, the test has been repeated 3 times.

As can be observed from the results, there is a qualitative difference in the response of the sensors when using DMMP compared to 2CEES. Specifically, in the case of DMMP, the relative counts for the vertical axis sensors (MOX A and PID A) show at the end of the measurement a value higher than the one recorded at the start of the data acquisition: such behaviour suggests that this time the local volume is still contaminated at the end of every 

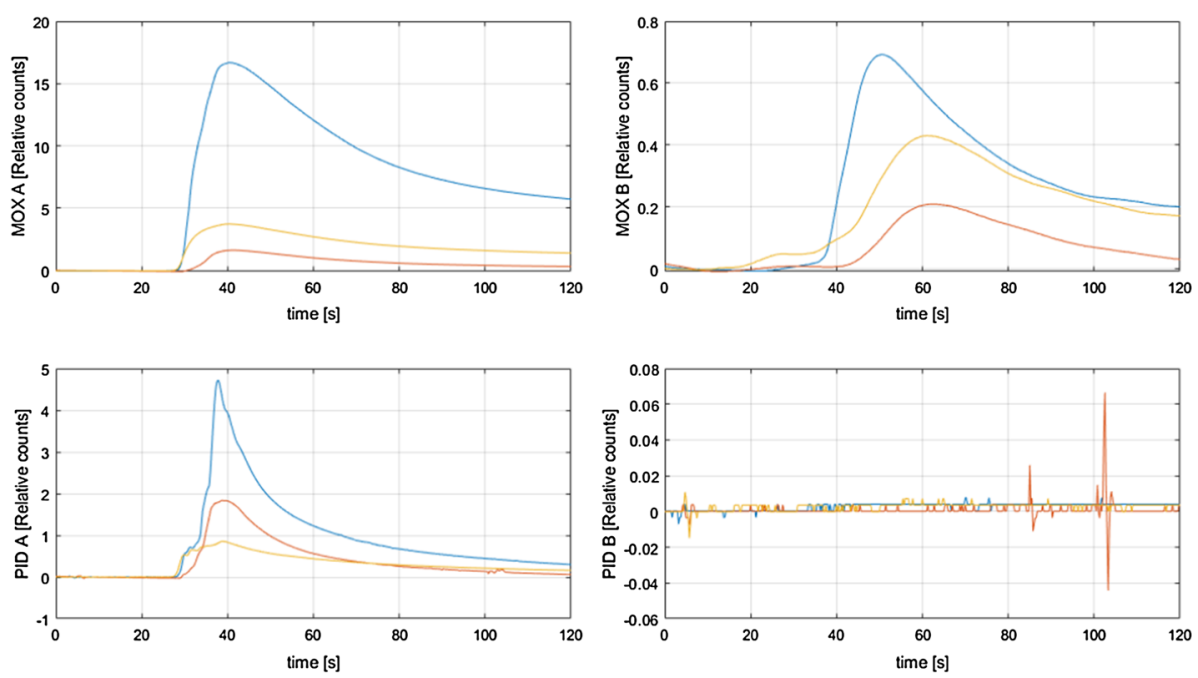

Fig. 12 MOX and PID response to the contamination with $100 \mu 1$ drop of DMMP (test repeated 3 times)
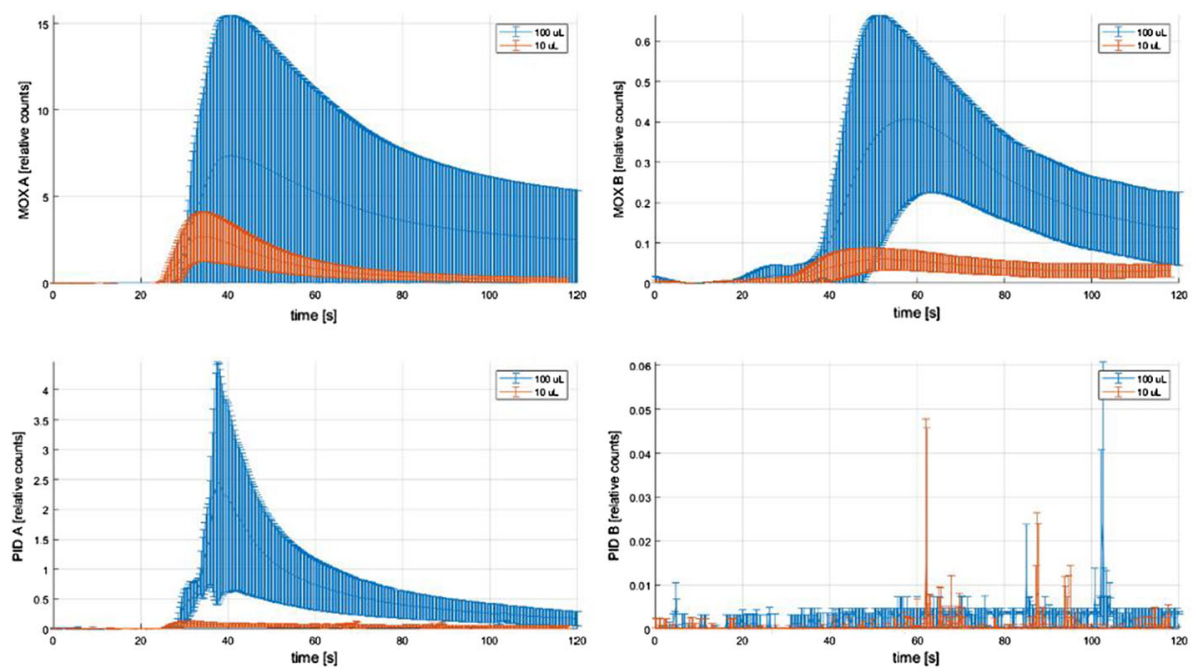

Fig. 13 MOX and PID overall responses with standard deviation to the contamination with $10 \mu 1$ and $100 \mu 1$ drop of DMMP

repetition and that every new acquisition started from a higher blank value. In Fig. 13 the overall relative counts for the two arrays of tests, showing the relevant standard deviation, are reported. The SD has been calculated, for each of the three arrays of tests (5 repetitions at $10 \mu \mathrm{l}$ and 3 repetitions at $100 \mu \mathrm{l}$ of agent volume), as the deviation from the arithmetic mean value of the measured counts, calculated at given intervals of time. 

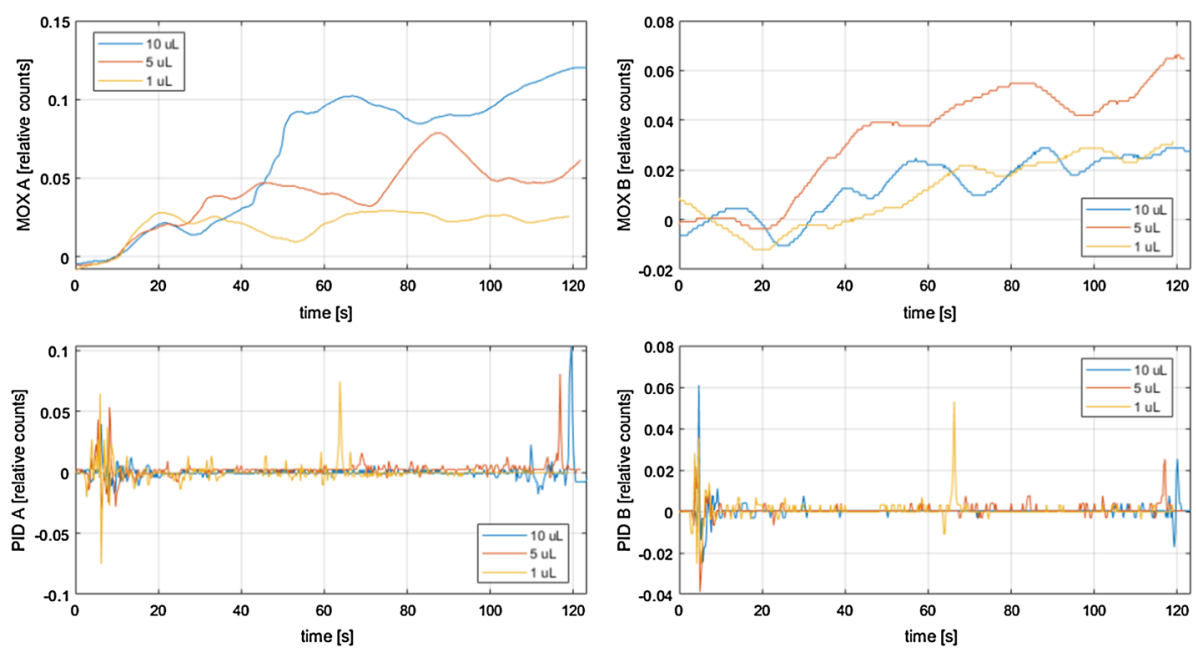

Fig. 14 MOX and PID responses to the contamination with $1 \mu 1,5 \mu l$ and $10 \mu l$ drop of PARAOXON

\subsection{Persistent nerve agent (tabun, GA) simulant tests}

Paraoxon has the aspect of an oily, slightly yellow liquid, and it is characterized by a faint fruit odour; it is weakly soluble in water $(3460 \mathrm{mg} / \mathrm{L})$.

Figure 14 shows the relative counts measured in response to the contamination obtained by pouring a certain volume, respectively, of $1 \mu 1,5 \mu 1$ and $10 \mu 1$, of paraoxon corresponding to a maximum local concentration of about $4 \mathrm{ppm}, 20 \mathrm{ppm}$ and $40 \mathrm{ppm}$. Sensor response has been analysed as described in the introduction of Sect. 4. As it can be observed, for MOX sensors the response resulted in an irregular increase in the number of counts during the acquisition time. This behaviour is possibly due to the long-lasting agent evaporation since the paraoxon is a strongly persistent agent which could result in a delayed and prolonged dissemination.

Regarding PID sensors, in both positions, no significant response was registered, possibly due to a too high IE of the contaminant concerning the PID lamp energy.

\subsection{Discussion}

From the measurements performed in the glove box, for both PID and MOX sensors, three different phenomena have been observed:

1. different responses to different chemicals;

2. different sensitivity at different background concentrations of MOX sensors;

3. different responses within the same set of tests of PID sensors.

The large difference observed in the response to chemical substances and their concentration is a standard characteristic of the MOX and PID sensor.

Looking at their technical datasheet, different MOX resistance-concentration responses can be found for different chemicals, while the density of the liquid and the molecular weight of the chemicals do not play a significant role (Fig. 15).

The different sensitivity at different concentrations is also explained by the sensor working principle. Figure 16 shows a typical sensor response characteristic [37]. 
Fig. 15 Sensitivity characteristics of TGS 2602 MOX sensor [36]

\section{Sensitivity Characteristics}

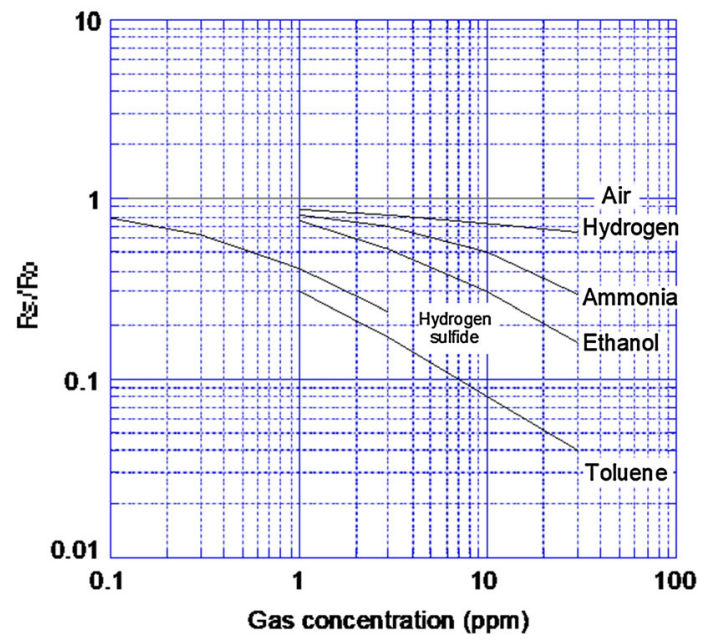

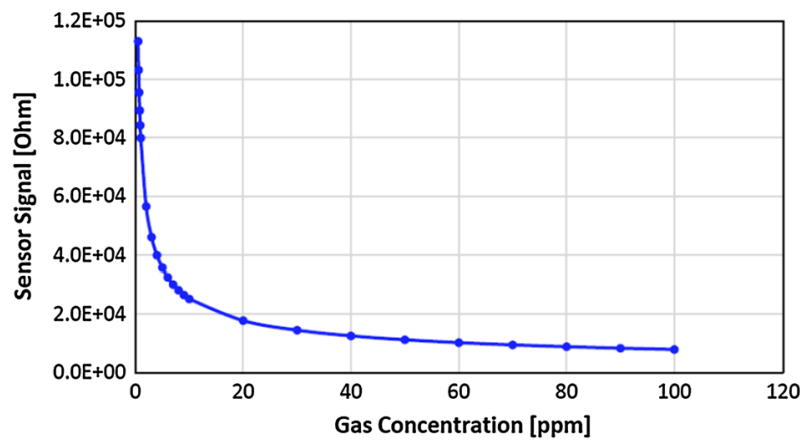

Fig. 16 Typical MOX response characteristics

The response Eq. (2) can be written as follows:

$$
R=A C^{-b}
$$

being the counts proportional to the inverse of the resistance; it follows (3):

$$
\text { Counts } \sim \frac{1}{R}=\frac{C^{b}}{A}
$$

where $A$ and $b$ are constants.

The increase of counts as a function of the increase of the concentration (4) can be calculated as:

$$
\frac{\mathrm{d} \text { Counts }}{\mathrm{d} C} \sim \frac{1}{A} C^{b-1}
$$

Being the $b$ constant smaller than 1 , the sensitivity decreases with the increase of the concentration.

Regarding PID sensors, the current signal produced is proportional to the number of ions generated from the PID lamp, which is, in turn, proportional to the lamplight absorbed 
Fig. 17 PID fraction of light absorbed vs concentration

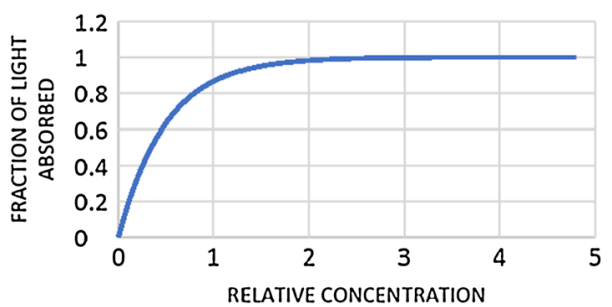

from the chemical agent; basically, the fraction of light absorbed increases with a growing concentration of the exposing substance. PID sensors response is influenced by the substance concentration following the Lambert-Beer equation [25] (5):

$$
I=I_{0} 10^{-\varepsilon l c}
$$

where $I$ is the intensity of light reaching the end of the sensor cavity, $I_{0}$ is the light intensity emitted from the lamp, $\varepsilon$ is the molar extinction coefficient characteristic of the substance, $l$ is the path length (sensor cavity depth) in length characteristic of the sensor, and $c$ is the agent the concentration.

While the fraction of light absorbed is (6):

$$
f_{a}=1-10^{-\varepsilon l c}
$$

This relationship, at low concentrations ( $\mathrm{ppb}$ to middle ppm range, which is in the order of magnitude of the concentrations used in this study), can be approximately considered linear, as it can be seen in Fig. 17, which shows the fraction of light absorbed $v s$ concentration of the agent. To summarize, in the case of low total absorbance $(\varepsilon l c<0.1), f_{a}$ can be approximated as linearly proportional to the concentration for a given substance ( $\varepsilon$ specified) and a certain sensor (specified $l$ ). At high concentrations, the substance completely absorbs the light and $f_{a}=1$, which means the sensor is saturated [25].

Within each set of tests characterized by the same amount of contaminant volume, it can be observed that different counts are reported. Although the response is globally proportional to the contamination and the followed procedure has been the same, possible narrow discrepancies are both due to human factor during the simulant deposition (lasting of pouring from the syringe, exact position on the heating plate, etc.) as well as to the turbulent flow of the hood conducted to expose the sensors to a different local concentration. This results in a high value of the standard deviation, as can be seen in Figs. 10 and 13, and is one of the cons intrinsically connected to the high sensitivity of MOX sensors, with which only qualitative answers can be given rather than quantifying contamination. Moreover, for PID A sensor the response is linearly proportional to the amount of pollutant in this range of concentration as per Lambert-Beer equation, while the number of counts registered by MOX A, as per its intrinsic characteristic (Figs. 15, 16), tends to a plateau with the increasing of contamination.

\section{Conclusions and Future Developments}

At a time in history when we are witnessing malicious actions and accidents involving TIC, VOC and CWAs, the main objective of this research was to develop a flexible, low-cost system, easily deployable in several scenarios. The integrated payload has been conceived for a variety of possible applications such as critical infrastructures and industrial contexts to 
avoid illegal discharge of poisonous substances or intentional release of toxic chemicals as weapons. By interfacing and intercommunicating different subsystems, the range and type of missions to be conducted can be expanded.

If the suspect or the alarm of an accident has been raised, by sending a drone equipped with this light, modular and plug-and-play feature, it is possible to assess whether a deviation from a clean baseline has occurred, without exposing personnel to unknown risks. With this tool, it is possible to give a first categorization of the class of chemical involved, to quantify the order of magnitude of the spread and to locate the point of origin of the release.

Regarding MOX sensors, experimental tests confirmed their high sensitivity and extremely low LOD, in the order of magnitude of ppb of VOC, have been detected in a confined environment, a characteristic that brings MOX sensors to be nowadays widely used as an electronic nose. As a result, the tests performed confirmed the aforementioned properties (high sensitivity, low weight, low power demand at an extremely low cost) and that those tools can be considered suitable for the application to UAVs.

The preliminary results coming from the tests conducted in laboratory under controlled conditions with the two detection techniques identified to be suitable for this integration-PID and MOX - have given encouraging results and have offered a good compromise between the several operational requirements pointed out, giving the basis for future experimental studies. The test campaign should be conducted by employing an array of MOX functionalized with different metal oxides in the way to perform qualitative discrimination of the substance, also with real CWAs. Further tests are planned to evaluate the influence of airflows generated by drone rotors on VOC detection, whether positive or negative.

The main application for which a single sensor or MOX sensor array could find a place on a drone is in an early warning detector. This would give an early warning if a certain number of counts multiple of the base value considered for a clean environment should be detected.

A new test campaign could be put in place to assess, for different susceptible areas, possible baseline values and their variation during the daytime and to optimize the desired value for a threshold alarm. This trigger could then activate a more sophisticated system, equipped with selective sensors and with a sampling system in the way to identify the nature of the substance responsible for the contamination. Indeed, foreseeing the inclusion of a sampling system on the UAV makes it possible to collect significant samples for subsequent laboratory analysis and confirmation of the preliminary collected results obtained from the survey flight. The chance to collect samples of the toxic compound implicated in the incident widens the possibility to confirm infield investigation with laboratory analysis [38].

Another key point to be investigated is the best location of the sensor on the UAV: this aspect has to be optimized, depending on the type of drone and on the geometry of the detection feature, to augment detection capabilities. A set of numerical fluid-dynamics simulations have already been conducted [17] and will execute a new set specifically dedicated to explore and understand the behaviour of rotary wings UAV shape and turbulence with a different chemical spread. This will allow proactive troubleshooting in advance to find out the viability of a configuration.

Acknowledgements We would like to thank the Joint NBC Defence School of Rieti and its staff for the availability of their structures and expertise in the conduct of the experimental tests and the Quantum Electronics and Plasma Physics Research Group for their technical support in the development activities. The authors are also deeply grateful to Dr. Luigi Di Landro for the generous help in the linguistic revision of the paper.

Author contributions D.D. and A.M. contributed to conceptualization; D.D. and P.G. carried out formal analysis; F.F. contributed to funding acquisition; F.F., A.C., R.R. and M.B. performed investigation; F.F., A.C., R.R., L.M. and M.B. were involved in experimental activities; P.G. and R.R. provided methodology; P.G. 
helped with project administration; D.D. carried out supervision; F.F. and A.M. performed writing-original draft; D.D., R.R., A.M. and F.E. performed writing-review and editing. All authors have read and agreed to the published version of the manuscript.

Funding This work has been partially funded by the Organization for Prohibition of Chemical Weapons (OPCW) with a grant received by the University of Rome Tor Vergata under the programme "Support for Research Programme” 2018 (Grant No. L/ICA/ICB/218790/19).

Availability of data and material The datasets generated during and analysed during the current study are not publicly available because they have been collected in a military centre, but are available from the corresponding author on reasonable request.

Code availability open source. Libraries, if necessary, are released under MIT, Apache and BSD license.

\section{Declarations}

Conflicts of interest The authors declare no conflict of interest.

\section{References}

1. M. Rossi, D. Brunelli, A. Adami, L. Lorenzelli, M. F. e F. Remondino, Gas-Drone: Portable gas sensing system on UAVs for gas leakage localization in Sensors, 2014 IEEE, Valencia, 2014

2. J. Nikolic, M. Burri, J. Rehder, S. Leutenegger, C. Huerzeler, R. Siegwart, A UAV system for inspection of industrial facilities. in IEEE Aerosp. Conf., 2013

3. A. Bachrach, S. Prentice, R. He, P. Henry, A. Huang, M. Krainin, D. Maturana, D. Fox, N. Roy, Estimation, planning, and mapping for autonomous flight using an RGB-D camera in GPS-denied environments. Int. J. Robot. Res. 31, 1320-1343 (2012)

4. L. Marconi, R. Naldi, L. Gentili, Modelling and control of a flying robot interacting with the environment. Automatica 47(12), 2571-2583 (2011)

5. M. Rossi, D. Brunelli, Autonomous gas detection and mapping with unmanned aerial vehicles. IEEE Trans. Instrum. Meas. 4, 65 (2015)

6. K. Rosser, K. Pavey, N. FitzGerald, A. Fatiaki, D. Neumann, D. Carr, B. Hanlon, J. Chahl, Autonomous chemical vapour detection by micro UAV. Remote Sens. 7, 16865-16882 (2015)

7. J. Karpowicz, Detecting radiological, biological and chemical threats with drones, 2016. Available: https:// www.commercialuavnews.com/public-safety/detecting-radiological-biological-chemical-threats-drones

8. G. Bolla, M. Casagrande, R. Dal Moro, M. Destro, ARIA: Air Pollutants Monitoring Using UAVs. in MetroAeroSpace, 2018

9. S. Bezantakos, F. Schmidt-Ott, B. Biskos, Performance evaluation of the cost-effective and lightweight Alphasense optical particle counter for use onboard unmanned aerial vehicles. Aerosol Sci. Technol. 52(4), 385-392 (2018)

10. Q. Gu, D. Michanowicz, C. Jia (2018) Developing a modular unmanned aerial vehicle (UAV) platform for air pollution profiling. Sensors 18

11. W. Marinelli, T. Schmit, J. Dupuis, P. Mulhall, P. Croteau, D. Manegold, M. Beshay, M. Lav, Cooperative use of standoff and UAV sensors for CBRNE detection. In Proceedings Volume 9455, Chemical, Biological, Radiological, Nuclear, and Explosives (CBRNE) Sensing XVI, 2015

12. F. Fumian, D. Di Giovanni, L. Martellucci, R. Rossi, P. Gaudio, Application of miniaturized sensors to unmanned aerial systems, a new pathway for the survey of polluted areas: preliminary results. Atmosphere 11, 471 (2020)

13. A. Rabajczyk, J. Zboina, M. Zielecka, R. Fellner, Monitoring of selected CBRN threats in the air in industrial areas with the use of unmanned aerial vehicles. Atmosphere 11, 1373 (2020)

14. J. Burgués, S. Marco (2020) Environmental chemical sensing using small drones: A review. Sci. Total Environ. 748 
15. M. Asad, O. Al Aidaros, R. Beg., M. Al Dhahri, S. Al Neyadi, M. Hussein, Development of Autonomous Drone for Gas Sensing Application. 2017 International Conference on Electrical and Computing Technologies and Applications (ICECTA), 2017

16. M. L. a. J. S. K. S. Do, The Effect of a Flow Field on Chemical Detection Performance of Quadrotor Drone, vol. 20, Sensor, 2020

17. F. Marturano, J.F. Ciparisse, A. Chierici, F. D’Errico, D. Di Giovanni, F. Fumian, R. Rossi, L. Martellucci, P. Gaudio, A. Malilzia, Enhancing radiation detection by drones through numerical fluid dynamics simulations. Sensors 6(1770), 20 (2020)

18. G. Korotcenkov, B. Cho, Metal oxide composites in conductometric gas sensors. Sens. Actuat. B Chem. 12, 117 (2016)

19. C. Wang, L. Yin, L. Zhang, D. Xiang, R. Gao, Metal oxide gas sensors: sensitivity and influencing factors. Sensors (Basel) 10(3), 2088-2106 (2010)

20. N. Masson, R. Piedrahita (2015) Approach for quantification of metal oxide type semiconductor gas sensors used for ambient air quality monitoring 208: 339-345

21. M. Rossi, D. Brunelli, Autonomous gas detection and mapping with unmanned aerial vehicles. IEEE Trans. Instrument. Measur. 65(4), 1-11 (2015)

22. D. Martinez, J. Burgués, S. Marco, Fast measurements with MOX sensors: a least-squares approach to blind deconvolution. Sensors (Basel) 19(18), 4029 (2019)

23. [Online]. Available: www.MSAsafety.com

24. K. A. Daum, M. Watrous, M. D. Neptune, D. I. Michael, K. J. Hull e J. D. Evans, Data for First Responder Use of Photoionization Detectors for Vapor Chemical Constituents, The INL is a U.S. Department of Energy National Laboratory (2006)

25. I. RAE Systems, The PID Handbook-Theory and Applications of Direct-Reading Photoionization Detectors (PIDs), San Jose, 2013

26. C.M. Zimmer, K. Kallis, F.J. Giebel, Micro-structured electron accelerator for the mobile gas ionization sensor technology. J. Sens. Sens. Syst. 4, 151-157 (2015)

27. Alphasense, AAN 305-06 VOC Correction Factor, Available: http://www.alphasense.com/index.php/ safety/application-notes/

28. RAESYSTEMS, Chemical-Warfare-Agent-Measurements-By-PID. Available: https://www.raesystems. $\mathrm{com} /$ sites/default/files/content/resources/Technical-Note-159_Chemical-Warfare-Agent-MeasurementsBy-PID_03-06.pdf

29. ELCOD project contribution 07 2019. Available: http://www.elcod.eu/wp/wp-content/uploads/2019/07/ 2019_elcod_5_pmeier.pdf

30. M. Raymond, Department of the Army, Memorandum Subject: Implementation Guidance Policy for New Airborne Exposure Limits for GB. GA, GD, GF, VX, H, HD, and HT 2004. Available: http://chppm-www. apgea.army.mil/chemicalagent/

31. Y. Sun, K. Y. Ong, Detection Technologies for Chemical Warfare Agents and Toxic Vapors, CRC Press, 2005

32. S. I. p. 1. D. NBC, Pub. N. 6210 Gli aggressivi chimici, Rieti: COMFORDOT, 2011

33. U. F. Aydogdu, Technological Dimensions of Defence Against Terrorism, NATO Science for Peace and Security Series-E: Human and Societal Dynamics, 2013

34. S. C. Biotechnology, Santa Crus Biotechnology 2020. Available https://www.scbt.com/browse/ chemicals/_N-18ovr15

35. S. Bartelt-Hunt, D. Knappe, M. Barlaz, A review of chemical warfare agent simulants for the study of environmental behavior. Crit. Rev. Environ. Sci. Technol. 38(2), 112-136 (2008)

36. F. Sensors, TGS2602 [Air Quality / VOC Sensor] 11 2020. Available https://www.figarosensor.com/ product/entry/tgs2602.html

37. R. E. Corporation, 2410 2017. Available https://www.idt.com/eu/en/document/dst/sgas711-datasheet

38. P. Pounds, D. Bersak, A. Dollar, Grasping from the air: Hovering capture and load stability. In IEEE Conf. Robotics Autom. ICRA, 2011 